\title{
Development of Smart Vehicle Security and Entertainment System (SSES) using Raspberry $\mathrm{Pi}$
}

\author{
Mujahid Tabassum, Mohab Gabr, Saju Mohanan, Kuruvilla Mathew
}

\begin{abstract}
This paper deals with development of a Vehicle Security and Entertainment System, which is being used to monitor, track the vehicle, and to offer local entertainment system. The development system makes used of two embedded devices to split the entertainment system from the security system to ensure isolation and security. The security system is equipped with camera, distress signal switch and GPS/GPRS module to track, report a problem, and monitor the vehicle by sending data to a centralized database server where vehicle owner can access and retrieve these data to guarantee the safety of the passengers and the vehicle too. The second system is the entertainment system, where this system uses a powerful Intel atom embedded device and local network to allow users to connect and offer entertaining services. These services include, E-Book library and multimedia streaming. The main concept of research to develop a low cost system to secure and entertain passengers on vehicles like buses, train and even cars. The development is cost effective and as well as can be modified to add extra modules or to develop extra entertainment services. If the vehicle is stolen the system is able to send a distress signal to the owner or company. They can help the passengers by monitoring through the vehicle camera. In this research we have successfully developed and tested the system.
\end{abstract}

Keywords: Vehicle Security; Entertainment System; Raspberry pi 2, Minnow Board MAX.

\section{T} here has been exponential sort advancement in communication engineering; more and more devices are becoming part of the network to offer flexibility to users. The transportation system need to have security and monitoring system to get benefited from developing technology. The current public transport sector lacks the technologies of the age. First issue with current transportation system is safety; in

Revised Manuscript Received on February 05, 2020.

* Correspondence Author

Mujahid Tabassum*, Department of Information Technology, Higher College of Technology, Muscat, Oman. E-mail: mujee1983@gmail.com

Mohab Gabr, Faulty of Engineering, Science and Computing, Swinburne University of Technology, Kuching, Sarawak, Malaysia. E-mail: 4239512@student.swinburne.edu.my

Saju Mohanan, Department of Information Technology, Higher College of Technology, Muscat, Oman. E-mail: saju.mohanan@hct.edu.om Swinburne University of Technology, Kuching, Sarawak, Malaysia. E-mail: kmathew@swinburne.edu.my

(C) The Authors. Published by Blue Eyes Intelligence Engineering and Sciences Publication (BEIESP). This is an open access article under the CC BY-NC-ND license (http://creativecommons.org/licenses/by-nc-nd/4.0/)

\section{INTRODUCTION}

Kuruvilla Mathew, Faulty of Engineering, Science and Computing,

3rd world countries like India, Pakistan or etc, people feel uncomfortable and avoid travelling by public transportations [1]. This is because of increasing crime rate especially in developing countries such as India, and Pakistan etc [1]. One of the terrifying incidence happened in the Delhi was a Bus Rape Case where a 23-year-old woman, Jyoti Singh Pandey was attacked by gang inside the bus, and got raped [1]. Another issue reported about safety was predicting fatal accidents by monitoring data for the vehicle including the position of the vehicle, and video from the camera [2]. One of the serious accident occurred in Pakistan, a fatal accident between a passenger bus, and an oil tanker near Karachi that, had left more than 60 dead [2]. By storing cameras data into storage or sending it to a server where it will defiantly help the police, and investigators to understand the cause of an accident or criminal act. Some other issues with public transportation are unreliability due to longer delay. Most of the public transportation like Buses has unpunctual timing where the vehicle might show up late. Furthermore, the vehicles that support online tracking are less accurate in specifying the real location. Another problem faced by long distance travel trip is that they easily getting tired, and bored. Passengers easily will get bored using public transportation, because it does not have any entertainment, or information system such as multimedia, and electronic library, which required a proper entertainment system.

In this research, we are proposing a Smart Security and Entertainment system (SSES) for vehicle tracking, monitoring and passenger entertainment. Currently, vehicle tracking and entertainment systems are very expensive. In this research we have designed and developed a cost effective, sustainable vehicle security and entertainment system. The SSES is accessible through a remote centralized web server through Internet. The vehicle owners and companies can login into the server through user name and password and view vehicle history and latest locations, signal strength, and distress signals of the driver. Besides that, the companies will be able to take live footages from the bus. The system allows users to track the vehicle current location and number of seats taken and estimated arrival time. In this paper we have explained our methodology, development process and testing parameters to ensure the reliability of the developed system. The proposed system aims to reduce passengers' tiredness by offering entertainment while travelling on the public transportations (e.g. buses) with affordable price. 
The developed system combines entertainment system and safety using small microcomputer that able to act as a server and able to host multimedia and readable materials which is stored on the system. Besides that, the system will be used to check the safety of the passengers and the status of the vehicle with real time monitoring system similar to the one used in airplanes (black box) in case of accidents, where it will keep the records from the cameras, and the location.

\section{LITERATURE REVIEW}

Currently, various types of similar products are available in the market but they are expensive and offer both services separately. For example, MYGPSTALK solution from TRAMIGO which cost around 940 RM for only tracking system alone, if we include entertainment system, it will become costly [3].

\section{A. Global Navigation Satellite System (GNSS)}

A satellite positioning system is a component assembly based on a constellation of artificial satellites capable of providing to a user through a portable small receiver that located position (longitude, latitude, and altitude), find object speed, and time. This system category repositioning is characterized by its high accuracy (few meters to current operational systems), its global reach, and compactness of stations. To meet specific needs (air traffic, vehicle tracking), several countries have developed regional scope of systems to increase reliability, and to further improve the accuracy available as DGPS -based ground stations or EGNOS using satellite signals in geostationary orbit.

The first satellite positioning system developed by the US first with TRANSIT for military use [4]. The Global Positioning System (GPS) became operational in 1995, which sets the operating principles set by the navigation satellite systems developed by other countries. The GPS system is based on a constellation of thirty satellites traveling on a medium earth orbit $(20,000 \mathrm{~km})$. This configuration allows a user, located an object or person on any point on the globe, the system functions with having at least four satellites in range. The user station calculates its position by determining the time taken by the signal emitted by each of the satellites which it knows the position by means of orbit parameters that it has transmitted to it. The accuracy of the result follows an extremely precise dating of the signal obtained by the carriage of atomic clocks on board satellites.

The USSR as a result of the United States develops its own system called GLONASS took place in 1996, after a period of the breakup of the Soviet Union, GLONASS has once again become operational in 2010 [4]. The Union European system with Galileo, and China with the system Beidou -2 (Compass) develop their own system, which should be fully operational in 2020 [5].

The use of stations is widespread with hundreds of millions of GPS sold every year to meet the needs of professionals, and the general public. The professional use include military, maritime navigation, air, and road, topography, and time synchronization. All satellite navigation systems provide different levels of service characterized by reliability, and / or a more or less accurately. All provide a basic service accessible for free, which is usually the one used by public. The stations can often exploit

the signals of multiple systems including GLONASS, and GPS. These passive systems can be complemented by radiolocation transmitters for logistic tracking applications including rescue, maritime traffic monitoring, and oceanographic research [4].

\section{B. Performance Augmentation System}

The existing satellite systems (GPS \& GLONASS) can be improved by so-called augmentation systems which deliver or overlay real-time corrections to increase accuracy as well as information ensuring the integrity of the corrections. The principle of these systems is that one or more ground stations continuously measure the error, and transmit a correction signal to users [6].

There are many different systems for different corrections provided to the receiver. Some systems transmit information on sources of error (clock deviations, ephemeris, and ionospheric delay), others provide the total observed deviation (differential), and others add information from the vehicle itself (speed, altitude, and etc.).

Generally classified these augmentation systems into three categories, depending on how the correction is calculated, and transmitted [6]:

Increase based on satellites: SBAS (Satellite-Based Augmentation System)

- WAAS (Wide Area Augmentation System) for the United States (CONUS service area called), complementing GPS.

- EGNOS (European Geostationary Navigation Overlay Service) for Europe (service area called ECAC), supplementing GPS \& GLONASS.

- MSAS under testing by Japan

Increase based on ground systems GBAS (Ground-Based Augmentation System): * DGPS (differential GPS).

- GAGAN being developed by India.

- Pseudolite (pseudo-satellite ground).

- Increase based on on-board systems (aircraft) ABAS (Aircraft Based Augmentation System).

- Inertial

These systems provide an accuracy up to centimeter. For air navigation, the ICAO request that the integrity of satellite navigation systems is monitored, and an alert is issued on board in case of loss of the integrity necessary (depending on the phase of flight).

\section{PROPOSED DESIGN AND METHODOLOGY}

In this research, we have designed and developed a secure and reliable vehicle tracking and entertainment system for public transportation that will help in improving public transportation safety and provide an entertainment service to the end users. We have performed unit test to check the performance of the systems, including network stress testing, database testing and to improve the implemented design. To evaluate and understand the functionality and feasibility of both systems we have run, tested and analyzed the results effectively. 


\section{A. System Components}

Figure 1 explain the design of our proposed system component design.

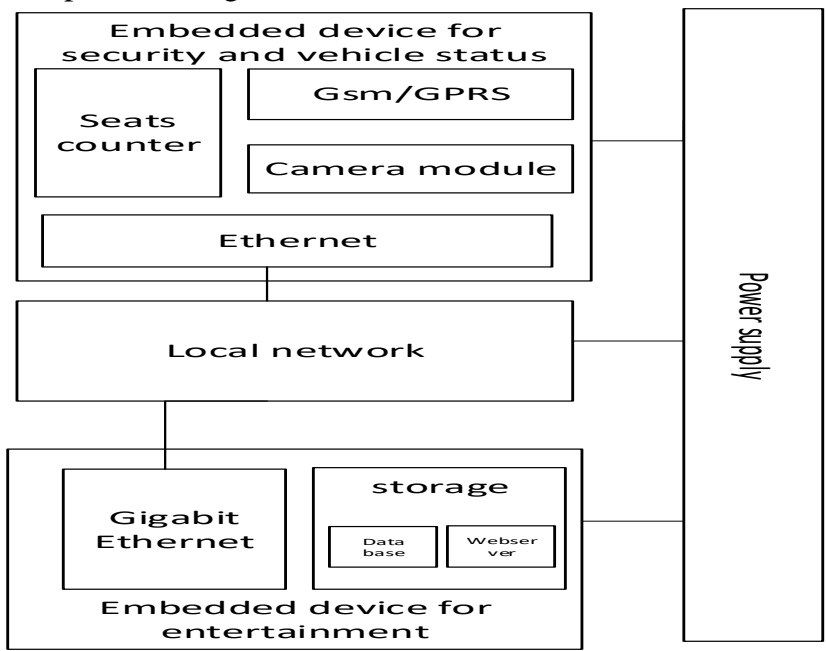

Fig. 1 Designed Entertainment System

Our developed system known as SSES that consist of two main systems:

- Vehicle Security and Tracking System: This system designed to manage the security related issues along extra services. It monitors all vehicle related services including location, signal strength, vehicle status (distress signal) and camera footages. It also monitors and keep track of passenger information. This service is used to monitor seats and number of passengers in a vehicle, to identify the empty and available seat number within a vehicle.

- Entertainment System: This system provides the as e-library and multimedia services.

B. Vehicle Security and Tracking System

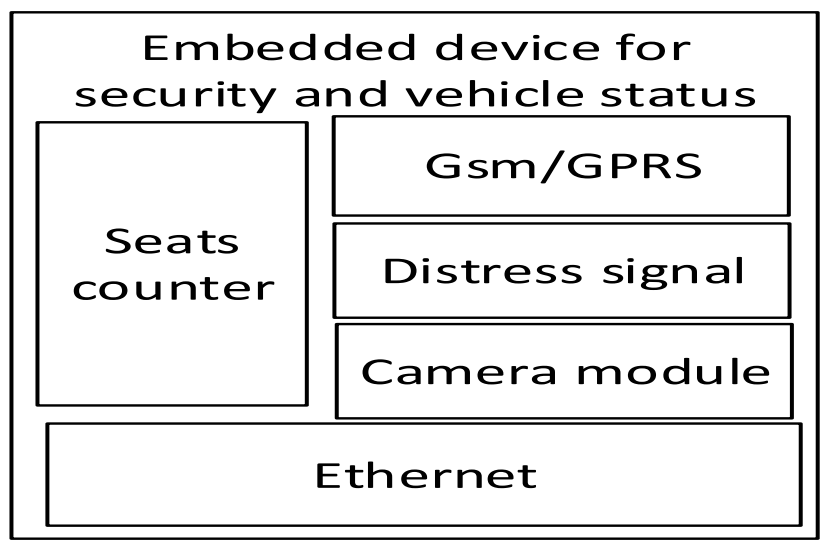

Fig. 2 Designed Security System

This system is fully cloud base and capable to monitor different vehicles. The system is connected to a centralized database server which stores instant information about vehicles like location, time stamp, speed, signal strength, and vehicle status in case of issuing distress signal. Camera is used to monitor the internal activity of vehicle and send the data to the respective server.

- Embedded Security Device: The embedded device which is used for the monitoring system is Raspberry pi 2. It runs with Broadcom BCM2836 Arm7 Quad Core Processor powered by Single Board Computer entertainment services for passengers on the vehicle such

running at $900 \mathrm{MHz}$, with $1 \mathrm{GP}$ RAM and CSI camera port for connecting the Raspberry Pi camera. The embedded device runs with Linux based system that starts the python code on starting up. The program will directly initialize and set the GPRS and GPS parameters. The raspberry pi is also used to take pictures and monitor the vehicle using its camera. Besides that, the embedded device has UART pins that can be used to transmit data to GPS/GPRS module. The pi is flexible where developers and engineers can implement and install any required sensors to monitor extra parameters for example, fuel monitoring, temperature sensing.

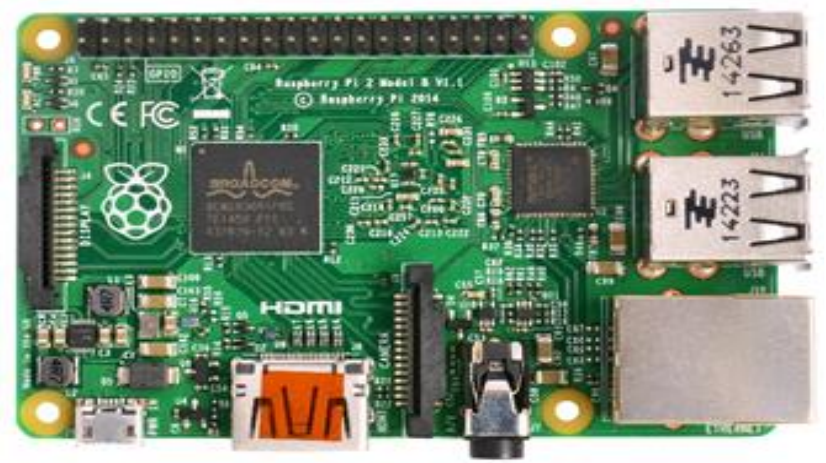

Fig. 3 Raspberry Pi 2 module

- GSM/GPRS Module: The module used is Sim908 GSM/GPRS module to collect information about location, speed and signal strength. This module board shipped with sim908.The Sim908 is a Quad-band GPS system which also support GPRS multi-slot class 10 . The GPS module supports 42 channel with high sensitivity (Tracking: $-160 \mathrm{dBm}$, Cold starts: $-143 \mathrm{dBm}$ ) and with accuracy of Horizontal position: <2.5m CEP [9]. This module can be controlled by AT commands. The module board has the following components:

1) Two power input interface: DC005 interface and a lithium battery interface.

2) Switch: It is used to open/close the input power supply for the module.

3) SMA antenna interface: there is a GSM antenna interface, and a GPS antenna interface onboard.

4) TTL serial interface: a GPRS TTL level interface and a GPS TTL level interface. This interface allowed communication with the module through the embedded device using AT commands.

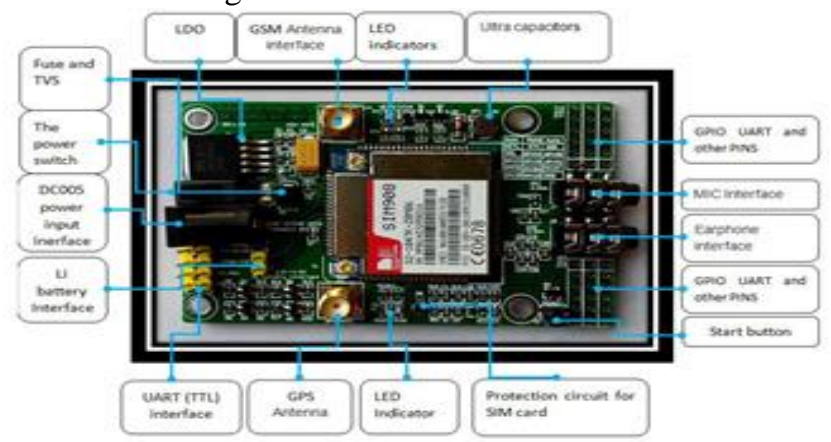

Fig. 4 GPS/GPRS Module [7]

Published By: 
- Seat Number Counter: The seat counting process can be done by various ways. One of the ways is to monitor using switches that are placed under the chair and in order to expand number of switches, the use of shift register is required. The second method to do seat counter is to place a tripod counter in front of the door using infrared. Finally, counting can be done by image processing of the passengers but with less accuracy. The first method is good for small numbers of passengers with great accuracy.

- Distress Signal: We have used a switch which issue distress signal and locate vehicle location and control by vehicle driver. This switch will change state into ' $S$ ' state reporting that immediately to the centralized controlling and management site. The vehicle manager/owner will be immediately identified to send maintenance team to the location.

- Raspberry Pi Camera Module: We have used a Camera Board Video Module which is a high definition camera module compatible with the Raspberry Pi. The module provides high sensitivity and low noise images. It uses Omnivision 5647 CMOS image sensor in a fixed-focus module with integral IR filter, and resolution of megapixel with maximum transfer rate 1080: 30fps.The camera uses 15-pin MIPI Camera Serial Interface (CSI-2).

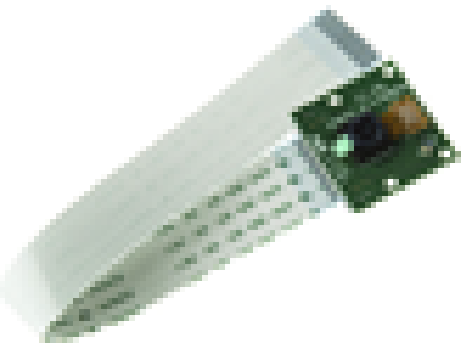

Fig. 5 Raspberry Pi Camera

\section{Entertainment System}

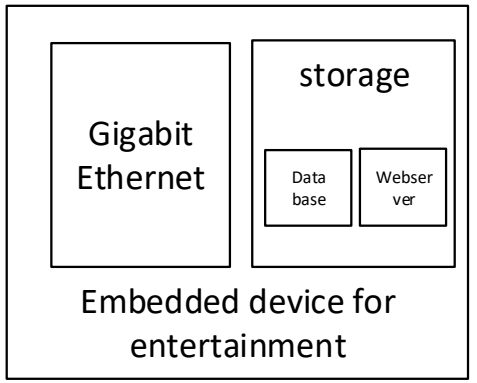

Fig. 6 Entertainment System Block

The entertainment system is a system designed to give special services to vehicle passengers. The main purpose is to entertain and motivate public transportation riders. The system takes use of a small embedded server with high processing power and connected to the vehicle's network. This system contains three main components.

- MinnowBoard Max: This embedded device is the heart of the entertainment system. The entertainment system uses MinnowBoard MAX which is an open hardware embedded board designed with the Intel Atom E38xx series SOC (known as Bay Trail). MinnowBoard MAX offers great performance, flexibility, openness, and standards for the price. The device equipped with 64-bit
Intel Atom dual-core processor with clocking speed of 1.33 Ghz. The used version of Minnow board max comes with 2 GB DDR3 Ram to handle multitasking efficiently. Besides that, it supports SATA2 with transfer speed of 3GB/sec. The board has Gigabit Ethernet which will help in handling multimedia streaming and other entertainment services.

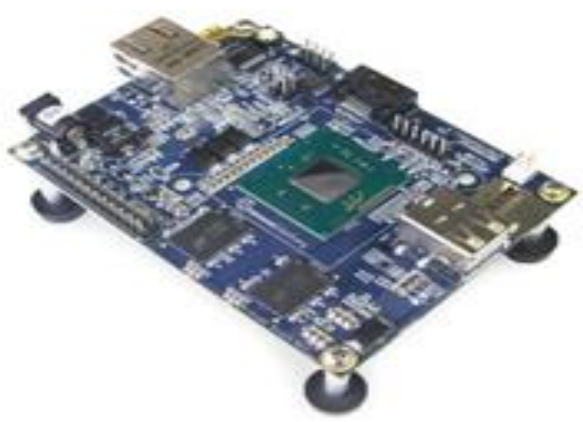

Fig. 7 MinnowwBoard

- Storage Sata2: The storage is important to run and install Ubuntu OS. The storage depends on the space required. The minimum space to run the operating system is 5 GB. Since our system will store multimedia and E-books, recommended to have a storage of at least $60 \mathrm{~GB}$. The 2.1-inch hard disk is sufficient but it is more vulnerable to mechanical failures due to having movable parts and subjects. The 2.1-inch hard drive could be replaced with Solid-state drive (SSD) which has higher life time and does not have moving parts to cause mechanical failures. Besides that, SSD consumes half to a third of the power of HDDs.

\section{IMPLEMENTATION}

The figure 8 show the network topology and overview of complete functional system.

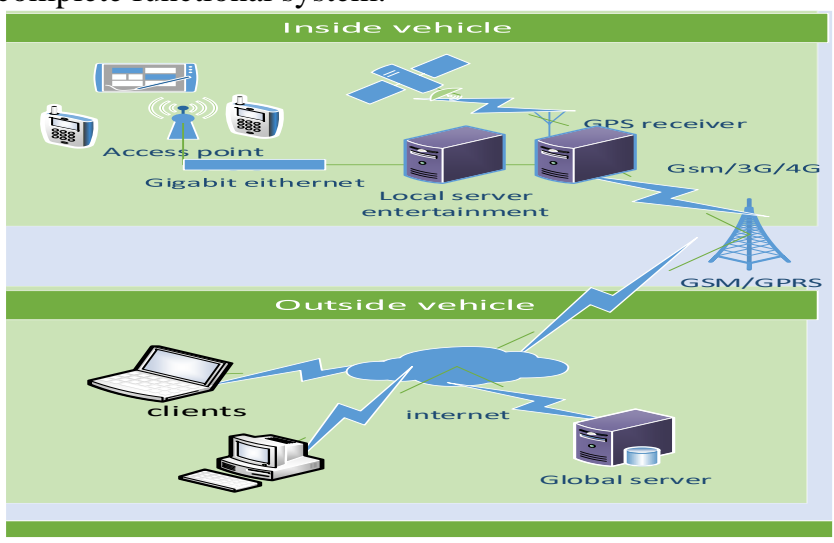

Fig. 8 Network Topology

The vehicle will be connected to two types of networks:

- Inside vehicle local network: This network designed to serve all the passengers and it is the medium to transmit entertainment system data. The network requirements and performance depends on how many users and how much the traffic generated by the entertainment server (MinnowBoard). 
- Outside vehicle network: This network includes communication between the remote server database and the local vehicle network. The communication is done through connecting both sides to the internet via GPRS module in the vehicle.

\section{A. Remote Server}

The remote server plays two roles which is database server or webserver for controlling and retrieving data from the database.

\section{B. Database Design}

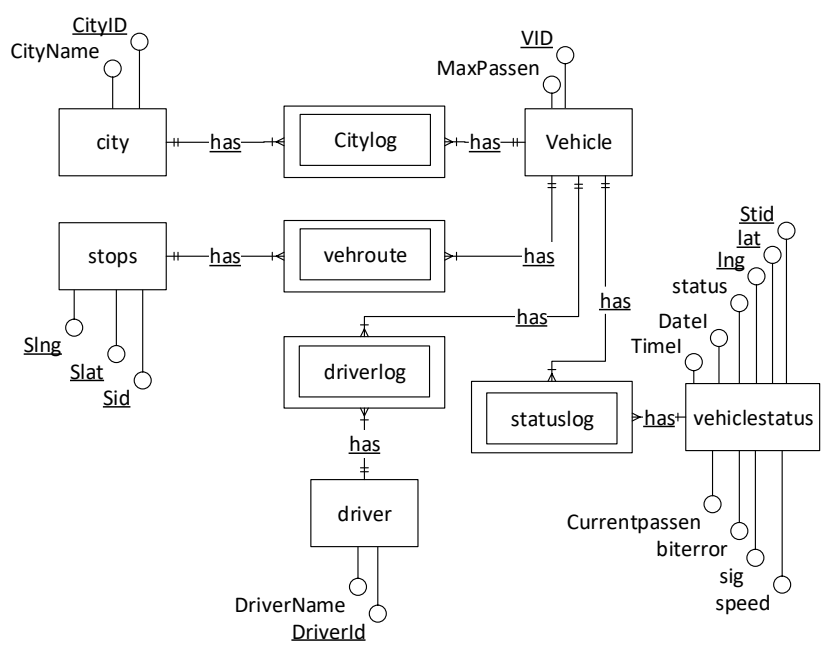

Fig. 9 Entity Relationship Diagram

The data base consists of nine entities such as Vehicle Entity (VID), City Entity (CityID), Stops Entity (stops), Driver Entity (DriverId), Vehiclestatus Entry. Citylog, vehroute, driverlog and statuslog entities are responsible to create full log and connect data to VID (vehicle identifier). The database capable of summarize everything related to certain vehicle like the city where the vehicle located, the station the bus stops at, the driver who drive the vehicle, and the vehicle status and information. The database implemented using MYSQL and the relation between entities followed the previous design. Where the weak entity borrows identifiers from the strong entities. For example, statuslog (weak entity) borrows stid, lat, lng from vehiclestatus entity and it borrows VID identifier to link all of the attributes related to certain vehicle (VID). In addition to that, these relations are helpful to eliminate database anomalies and guarantees consistency.

\section{Web Server}

This webserver has two categories: General user version, and administrator.

General user allowed to see real-time tracking of a certain vehicle based on its VID. The users are able to see the estimated reach time and number of the current passengers.

The administrator has extra features compared to general user like reviewing vehicles history status (reviewing the log, speed, signal strength, distress signal, bit-error rate), and live footages from the cameras.

\section{Google Map API}

The web server displays real time information for the selected vehicle by using JavaScript that load the data from the generated xml file to avoid direct user interaction with the database. The google api is used to locate the vehicle and display the information into information window. The marker on the map will be updated every $250 \mathrm{~ms}$ for smooth real-time tracking. IN addition to that, this api can estimate time based on road statistics and maximum speed. The statistical arrival time estimation accuracy is sufficient, since, vehicle has a lot of uncertainty (e.g. the vehicle stops, or traffic jam).

\section{RESULT ANALYSIS AND TESTING}

\section{A. Security Monitoring System}

We have successfully tested the developed system security components including GPS, map location, monitoring camera and distress signal. The GPS module test shows that in order for the GPS to get fixed location for first running, it needs about 30 seconds (cold start). The GPS tracking sensitivity about $-143 \mathrm{dBm}$ and consumes $76 \mathrm{~mA}$ for tracking $[4,6]$. The GPS maximum delay can reach to one second which is acceptable for real-time tracking locations, the main reason for that is the delay of transmitting and receiving the packets in the webserver.

The camera used Pi camera module, which can be added as an extra component for the real-time monitoring. In order to run the camera smoothly with high resolution, 4G module should be added and redirection for external requests should be forwarded to the Raspberry PI camera webserver. The web interface has many settings to reduce the traffic that consumed by recording videos. Thus, to reach the lowest consumption of bandwidth, the camera has to run on low resolution and with less frames per seconds.

The distress signal uses an interrupt that changes the status of the vehicle and send it to the server database immediately.

\section{B. Entertainment System}

We have successfully tested the entertainment system including the performance of the local server and the network maximum performance and processing over the system. The first part of the entertainment system is about electronic book store, where users can get some free E-books. The eBook library uses Joomla content management. Joomla was used to manage the content freely and easily with some powerful add-ons for e-library.

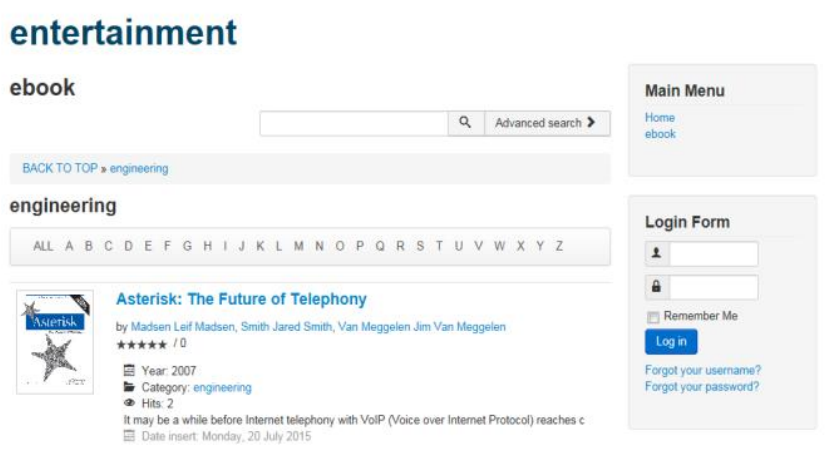

Fig. 10 E-Book Accessibility

Published By:

Retrieval Number: C5932029320/2020@BEIESP




Before implementing multimedia website, verifying the network and the microcomputer capacity too. The multimedia store can be added to Joomla too. Joomla is a content management system (CMS), which enables user to build Web sites and powerful online applications. Many aspects, including its ease-of-use and extensibility, have made Joomla the most popular Web site software available. Joomla is an open source solution that is freely available to everyone [8].

Extensible Markup Language (XML) is a markup language that defines a set of rules for encoding documents in a format which is both human-readable and machine-readable. It is defined by the W3C's XML 1.0 Specification [9] and by several other related specifications, [10] all of which are free open standards [11].

The design goals of XML emphasize simplicity, generality and usability across the Internet. [12] It is a textual data format with strong support via Unicode for different human languages. Although the design of XML focuses on documents, it is widely used for the representation of arbitrary data structures [13] such as those used in web services.

Our results show that around 7934 Mbytes of selected data which represent $87 \%$ of the total data queries. That number are significantly huge because one user managed to consume a lot of data and bandwidth. The database direct interaction to get last location coordinate will consume a lot of the server resources especially in future when the database become a humongous and full of data. We have observed that each user requested for temporary information from the database 4 times a second which has generated estimated 183 Megabytes, imagine having more than one user (e.g. 1000) or users accessing more than one tap the same page. Therefore, we had to come with a solution eliminate unnecessary interaction with the database by using XML file.

The xml file will reduce data processing and bandwidth of loading unchanged data many times, the xml file will only store the latest data directly after sending it from the vehicle tracking system. Therefore, reduction of data loading on the SQL server and store only one entity. The system still need SQL database to record everything with time and it is easier in processing big data like showing all coordinate in certain date.

\section{Network Performance}

The network performance is the most critical component in the entertainment system where we have to find the maximum bandwidth that the server can offer. Iperf3 tool was used for stress testing via flooding the server with packets and processing these packets.

First of all, we have tested the maximum throughput using Point-to-point Gigabit connection.

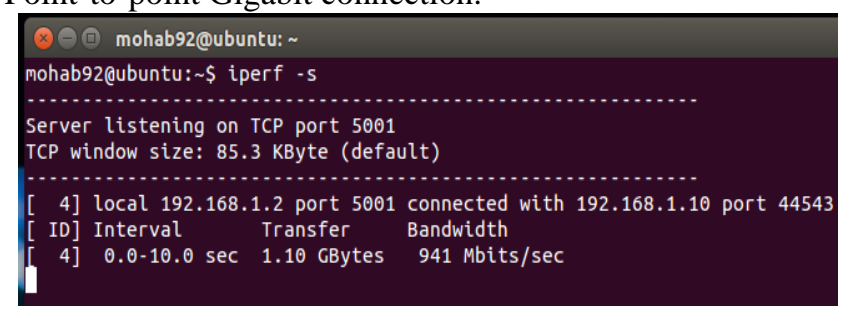

Fig. 11 Gigabit Direct Connect

Figure 11 shows that the maximum real bandwidth offered by the Gigabit Ethernet on entertainment server is about 941 Mbit/second from one client to the server. The performance bandwidth performance of the network interface controller is about $91.89 \%$ of the theoretical performance which is acceptable for point-to-point access point connectivity.

The figures 12 and 13 shows the increasing number of users connected to the server.



Fig. 12 Three client communication

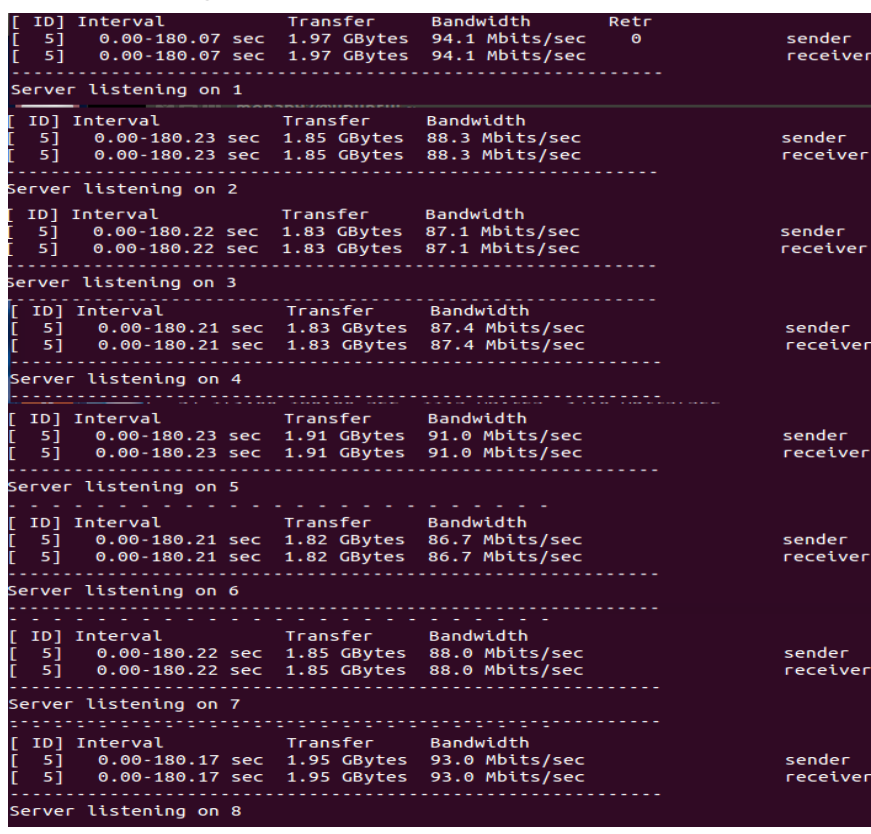

Fig. 13 Eight client communication

By increasing number of simultaneously running clients, we were able to predict and estimate the bandwidth with certain number of users.

The UDP performance is better than TCP specially in streaming videos. The percentage successful transmitted packets are $86 \%$ with a bandwidth performance of $93.55 \%$.

\begin{tabular}{|c|c|c|c|}
\hline [ID] Interval & Transfer Bandwidth & Jitter & Lost/Total Datagrams \\
\hline [ 5] $0.00-180.06 \mathrm{sec}$ & 20.1 GBytes $958 \mathrm{Mbits} / \mathrm{s}$ & $0.128 \mathrm{~ms}$ & $377024 / 2631490(14 \%)$ \\
\hline
\end{tabular}

Fig. 14 Bandwidth and Lost

Published By:

Retrieval Number: C5932029320/2020@BEIESP DOI: 10.35940/ijeat.C5932.029320 Journal Website: www.ijeat.org 


\section{CONCLUSION}

In conclusion, the entertainment system and the security are feasible to implement in public and private transportation vehicles. The security system can be improved by cutting direct interacting with the normal users by storing latest updates on the file instead of going through big data and sending big query string. Besides that, the security system needs a durable covering that is durable and can handle accidents. In addition to physical protection, the camera module needs high speed internet connectivity like 4G connection because GPRS is not enough to transmit live streaming camera. The entertainment system is capable of running simple website with video buffering and e-book store for 20-30 devices, where each user is allocated maximum 20 $\mathrm{Mbit} / \mathrm{second}$ and that result is the maximum throughput of the server and to achieve that using wireless connectivity a powerful access point must be used. This whole system was successfully developed and tested. This is a low cost system, therefore, companies and government bodies can easily adopt to enhance passenger safety and offer them entertainment when travelling on the local busses.

\section{REFERENCES}

1. Hugo, G. and Suzannah, H., 2013. “She's as Guilty as Her Rapists': Fury as Indian Guru Blames Tragic Student, 23, for the Vicious Gang Attack Which Killed Her". Mail Online.

2. BBC, 2014. India gang rapes: "police says all suspects arrested". BBC news, p. 1.

3. Tramigo, [Accessed: 10- Nov- 2019].

4. Tsui, J.B.Y., 2005. Fundamentals of global positioning system receivers: a software approach (Vol. 173). John Wiley \& Sons.

5. Ligthart, L.P. and Prasad, R. eds., 2013. Communications, Navigation, Sensing and Services (CONASENSE). River Publishers.

6. Leick, A., Rapoport, L. and Tatarnikov, D., 2015. GPS satellite surveying. John Wiley \& Sons.

7. SIM908_Hardware Design_V1.03, 1st ed. China: SIMCom Wireless Solutions Ltd, 2010.

8. A. Kempkens, 'About Joomla!', Joomla, 2015. [Online]. Available: https://www.joomla.org/about-joomla.html. [Accessed: 11- Nov- 2015].

9. XML 1.0 Specification". World Wide Web Consortium. [Accessed: 11Dec- 2019].

10. Bikakis N., Tsinaraki C., Gioldasis N., Stavrakantonakis I., Christodoulakis S.: "The XML and Semantic Web Worlds: Technologies, Interoperability and Integration. A survey of the State of the Art" In Semantic Hyper/Multi-media Adaptation: Schemes and Applications, Springer 2013.

11. W3.org, 'W3C Document License', 2015. [Online]. Available: http://www.w3.org/Consortium/Legal/2002/copyright-documents-2002 1231. [Accessed: 10- Nov- 2019].

12. W3.org, 'Extensible Markup Language (XML) 1.0 (Fifth Edition)', 2015. [Online]. Available: http://www.w3.org/TR/REC-xml/\#sec-origin-goals. [Accessed: 10Nov- 2019].

13. P. Fennell, 'Extremes of XML', XML London 2013 Conference Proceedings, 2013.

\section{AUTHORS PROFILE}

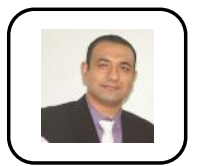

Mujahid Tabassum is a lecturer in Higher College of Technology, Muscat, Oman. He obtained a Master of Science (Computer Engineering) degree from the Halmstad University, Sweden. Currently, pursuing PhD from Malaysia. He is a Chartered Engineer and having more than 10 years of teaching and research experience. He is a member of IEEE, IET, ACS, MBOT and IAENG. His research interests are IoT, Wireless Sensor Network, Computer Networks and Artificial Intelligence.

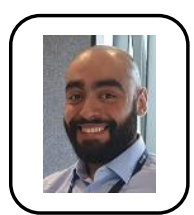

Mohab Gabr is a Network Integration Engineer at nbn ${ }^{\mathrm{TM}}$ Australia. He graduated from Swinburne University of Technology, (Sarawak Campus), Australia with a Telecommunication and Network Engineering degree.

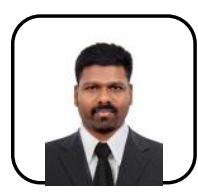

Saju Mohanan is an Indian-born researcher and academician, working with Department of Information Technology, Higher College of Technology. He is a PhD holder in Computer Science and Engineering and has more than 10 years of teaching and research experience. $\mathrm{He}$ is an active researcher with dynamic capabilities, hardworking and enthusiastic to learn advanced technologies, and to make use of technological benefits to differen stakeholders and he is keen to explore better possibilities from the current innovations.

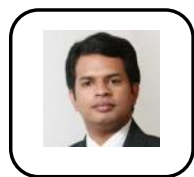

Kuruvilla Mathew holds a $\mathrm{PhD}$ degree from Unimas, Malaysia. He has more than 12 years of teaching experience. His research interests are Computer Networks, Wireless Sensor Networks and Security. He is a senior member of IEEE. 